\title{
Injury pattern in lethal motorbikes-pedestrian collisions, in the area of Barcelona, Spain.
}

\begin{abstract}
Introduction: There are several studies about M1 type vehicle-pedestrian collision injury pattern, and based on them, there has been several changes in automobiles for pedestrian protection. However, the lack of sufficient studies about injury pattern in motorbikes-pedestrian collisions leads to a lack of optimization design of these vehicles. The objective of this research is to study the injury pattern of pedestrians involved in collisions with motorized two-wheeled vehicles.
\end{abstract}

Methods: A retrospective descriptive study of pedestrian's deaths after collisions with motorcycles in an urban area, like Barcelona was performed. The cases were collected from the Forensic Pathology Service database of the Institute of Legal Medicine of Catalonia. The selected cases were categorized as pedestrian-motorcycle collision, between January $1^{\text {st }} 2005$ and December $31^{\text {th }}$ 2014. Data were collected from the autopsy, medical, and police report. The collected information was then analyzed using Microsoft Excel statistical functions.

Results: Traumatic Brain Injury is the main cause of death in pedestrian hit by motorized two-wheeled vehicles (62.85\%). The most frequent injury was the subarachnoid hemorrhage, in $71.4 \%$ of cases, followed by cerebral contusions and skull base fractures (65.7\%). By contrast, pelvic fractures and tibia fractures only appeared in $28.6 \%$.

Conclusions: The study characterizes the injury pattern of pedestrians involved in a collision with motorized two-wheeled vehicles in an urban area, like Barcelona, which has been found to be different from other vehicle-pedestrian collisions, with a higher incidence of brain injuries and minor frequency of lower extremities fractures in pelvis, tibia and fibula.

\section{Key words}

Pedestrian collision, injury pattern, motorcycles, motorbikes, autopsy, traumatic brain injury. 


\section{Introduction}

Traffic accidents, including pedestrian-vehicle collisions, are one of the leading causes of death in developed and developing countries. In 2005 in the United States more than 64,000 of the injured people were pedestrians ${ }^{1}$, in Germany in 2008, a total of 695 pedestrians were killed and 33.733 were injured ${ }^{2}$. The magnitude of the epidemiologic problem takes even more importance in developing countries, for instance in Ghana $60 \%$ of people who died by traffic accidents were pedestrians ${ }^{3}$. Therefore, there are many initiatives undertaken by different institutions to reduce the number of accidents and fatalities involving pedestrians, as well as the severity of the sustained injuries.

In recent years there has been greater awareness in relation to the so-called vulnerable users on the road such as pedestrians, cyclists and two-wheeled motor users $^{4,5}$. The highest incidence of motorcycle accidents are related to the characteristics of the vehicle, such as its high maneuverability or its great power in relation to its weight. Moreover, its high morbidity and mortality are due to both the driver and the passenger due to the lack of body structure and protection in comparison to other vehicles. Additionally, it particularly affects young people, leading to a great loss of labor capacity or loss in life years ${ }^{6}$.

On the other hand, in relation to pedestrians, awareness is such that the European Union Directive 2003/102/EC of the European Parliament and of the Council from November $17^{\text {th }} 2003$ related to the protection of pedestrians, amending Directive 70/156 / EEC, has forced the automotive industry to redesign M1 vehicles for their design to be more friendly for the pedestrians in case of collision. This regulation is not intended to avoid any injury in case of impact, but to minimize its consequences in order to prevent unnecessary injuries. These adopted measures consist, inter alia, to increase the distance between the hood and the rigid structures, to include collapsible structures designed to absorb energy, to incorporate energy absorbing elements between the pedestrian and rigid structures, reducing the potential of area being impacted, etc. ${ }^{\text {. }}$

Most of the current research has been done on type M1 vehicles. Currently there is a lack of knowledge of the injury patterns in other vehicles such as motorcycles. Is there any difference between M1 injury pattern and motor vehicles with two wheels? Is vulnerability affected by geometric factors, stiffness, size, speed, momentum or a combination of all of them? ${ }^{8}$. It would seem common sense that stiffness, speed and mass are against the pedestrian, since in this type of collision pedestrians are injured in 
different levels of severity or, as we will discuss in this research, even death. However, there is not legislation for the design of motorcycles to try to minimize the consequences of a pedestrian impact.

It is not difficult to find literature on motorcycle accidents and injuries sustained by the drivers, or about the pedestrian injury pattern from vehicle collisions ${ }^{1,2}$, however it is challenging to find literature relative to collisions of pedestrians struck by motorcycles. The aim of this study is to describe the injury pattern of pedestrians who have been struck by motorcycles that resulted on death, in urban areas.

\section{Method}

A retrospective descriptive study of pedestrian's deaths after collisions with motorcycles in an urban area like Barcelona was conducted between January $1^{\text {st }} 2005$ and December $31^{\text {th }} 2014$. The cases were collected from the entire registry of the Forensic Pathology Service database of the Institute of Legal Medicine of Catalonia. The inclusion criterion was to be categorized as pedestrian-motorcycle collision. The data were collected from the autopsy report and complemented with the medical and police files. When the pedestrian was deceased in the site of the accident, a copy of the ambulance assistance report was investigated. In cases that required transfer to a hospital, data from the final medical report were also investigated.

The Pathology Service of the Institute of Legal Medicine of Catalonia performed a total of 12797 autopsies in the studied period of time, from those total cases 837 were categorized as motor vehicle accidents and 300 were pedestrians involved in collisions with any type of vehicle. A total of 38 pedestrians were categorized in the data base as pedestrian's deaths after collisions with motorcycles. After double checking the autopsy reports, medical and police reports three cases were excluded for not meeting the inclusion criteria, in two cases for inaccurate codification and one for fatality not related to the motorcycle collision. The total pedestrians analyzed in this study were $n=35$.

Data were collected and analyzed with Microsoft Excel statistical functions. Victim's data collected were: age, sex, date of accident, date of death, hospital admission, cause of death; and injuries sustained on head, neck, upper and lower extremities, thorax, abdominal and back. For the interpretation of the injuries the Abbreviated Injury Score (AIS) was used for each reported injury, and from these scores the Maximum Abbreviated Injury Score (MAIS) of head, neck, upper extremities, lower extremities, 
thorax, abdomen, back and general was calculated. Additionally the Injury Severity Score (ISS) (sum of the squares of the highest AIS score in the three most severely injured body regions) and the New Injury Severity Score (NISS) (the sum of the squares of the highest AIS score regardless of the body region in which they occur) were calculated.

\section{Results}

A total of 35 pedestrian fatal cases were collected, 18 men and 17 women, with an average age of $67.4 \pm 22.7$ years for women and $72.3 \pm 15.2$ years for men.

From the 35 pedestrians, $91.4 \%(n=32)$ of the cases received hospital treatment while the other three people died in the crash site. Those who received hospital care, $80 \%$ died the same day of the accident or the next day; and those who survived beyond two days had an average hospital stay of 4 days. The results are presented in Table 1.

Please, Insert Table 1

The most important cause of death was Traumatic Brain Injury (TBI) in $62.9 \%$ of the cases, followed by hypovolemic shock in $20 \%$ of the cases, and a combined thoracic and brain trauma in $5.7 \%$ of the cases (Table 2).

Please, Insert Table 2

When the cause of death reported in the autopsy was TBI, the MAIS was the punctuation of the head (except in two cases, cases 11 and 16, where the cause of death was the TBI but had an equal MAIS for head and thorax) whereas when the cause of death was the hemorrhage the MAIS was the one found in lower extremities, thorax and / or abdomen.

The pedestrians died from injuries sustained in the accident in all the cases, except in one of the cases, whose cause of death was pneumonia as a complication of injuries after a long hospital stay.

The $31.4 \%$ of patients sustained an AIS 5 on the head and $91.43 \%$ sustained severe injuries with AIS $3+$ in this anatomical region. The next often anatomical regions injuries with an AIS 3+ were the thorax (45.7\%) and the lower extremities (20\%). 
According to the MAIS, $40 \%$ had a MAIS $5+$ and $60 \%$ had a MAIS $4+$, and only one case $(2.85 \%)$ was coded as MAIS 6 due to a thoracic aortic rupture.

The average calculated ISS was $27.7 \pm 13.4$. The ISS median was 27 , with data between 9 and 75. A significant variation was observed regarding the NISS, with an average of $36.2 \pm 13.9$ and a median of 34 , with data also between 9 and 75 (Table 2). It should be noted that pedestrians with highest scoring both at ISS and NISS presented the most serious injuries in thoracic and abdominal areas.

Using additionally the AIS categories 9-15 for moderately injured patients, 16-24 for severely injured patients and 25-75 for critically injured patients, a total of 5 pedestrians were included in the 9-15 (14\%), 9 included in the 16-24 (26\%) and 21 pedestrians in the category of critically injured patients $25-75(60 \%)$.

The main injuries grouped by anatomic region can be observed in the Table 3 .

Please, Insert Table 3

The most frequent injuries found (Table 3) were derived from $\mathrm{TBI}$, specifically subarachnoid hemorrhage in $71.4 \%$ of cases, followed by cerebral contusions and skull base fractures (65.7\%) and subdural hematomas (54.3\%).

The most common thoracic injuries were rib fractures, found in $45.7 \%$ of the cases while the most frequent abdominal injuries were liver contusion/laceration and mesentery contusions, found in $17.1 \%$ of the cases.

The most frequent injuries in lower extremities where pelvic and tibia fractures, found both in $28.6 \%$ of the cases; fibula fractures were found in $20 \%$ of the cases.

In the neck, back and the upper extremities the frequency of the sustained injuries was lower than $10 \%$, with the exception of the thoracic vertebrae fracture which was found in $14.3 \%$ of the cases.

\section{Discussion}

Although Legal Medicine ${ }^{9}$ has always considered the car pedestrian collision as typical or atypical, according to four phases (crashing, droping, dragging and crushing), recent literature has indicated that this classification is not completely satisfactory. In practice, researchers consider an important distinction between first and second impacts ${ }^{10}$ : the 
first impact is the one against the vehicle, and the second impact is the one against the floor.

Both classifications are based on injuries suffered by pedestrians after a collision with vehicles of four wheels; but the kinematics of the collision as well as the injury pattern is different between two-wheeled vehicles and four-wheel vehicles.

The main contribution presented in this study is that it focuses on the pedestrian injury pattern struck by two wheels vehicles, since other research has been studying the injury pattern of the accidents in general, but not specifically to the ones involving pedestrians hit by motorcycles, or at least they don't distinguish between vehicles ${ }^{1,2,11}$, 12.

In our study, as in most studies on accidents $1,3,11,12$, most people hit by a car were male, as contrast with the study of Ehrlich et al. ${ }^{2}$ where a predominance of female were found.

Our mean age was much higher than that of other studies, such as Peng et al. ${ }^{12}$ with $27.6 \pm 0.3$ years or the one of Schmucker et al. ${ }^{2}$ with 37.1 years. In the study of Ehrlich et al. ${ }^{11}$, the age average for women was similar to our study, 69 years on average, with cases reported between 16 and 91 years, and in our study $67.4 \pm 22.7$ years on average with cases from 16 to 92 years.

Most of the injured died in hospital (91.4\%). Those who survived beyond 24 hours, had a median stay of 4 days, which coincides with the median of hospital stay in the study by Starnes et al. ${ }^{1}$

In the present study, the most common cause of death was the TBI, a fact that coincides with the study Schmucker et al. $^{2}$ In both studies, the most common injuries were subarachnoid hemorrhage and brain contusions. In our case, this may be because the cases studied are fatalities and the injuries of higher mortality are those located on the head. In other studies present in the literature the most frequent injuries were in muscle-skeletal ${ }^{12}$ system, above all in lower extremities, not only in developed countries, as the United States of America ${ }^{1,11}$ or European ${ }^{14}$ countries but also in developing countries such as India ${ }^{15}$. This could be explained because our data were collected from forensic data not from hospital reports like in these studies.

In lower limb injuries, the most common fractures were the pelvis (28.57\%), this is more frequent than in the study of Siram et al. ${ }^{11}$ where they represent $9.2 \%$, or in the 
study performed by Starnes et al. ${ }^{1}$ where they represent $14.4 \%$, which also reflected a higher incidence of pelvic fractures in women than in men. In our case pelvis fracture appears in 10 cases out of 35, in 6 women and 4 men. The higher frequency may be due to the location of the vehicle with which the collision occurs. In accidents with utility vehicles, pelvic fractures usually occur when the pelvis hits against the hood ${ }^{7}$, in the wrap around the vehicle, resulting in a reduction in collision speed and a greater area of impact, therefore reducing the pelvic load. In contrast, in the case of accidents for two-wheeled vehicles, the collision occurs in the front area of the vehicle so that there is a direct impact against the pelvic area, without the speed reduction; and due to the small surface of contact this causes stress concentration to the pelvic area. This could explain both the increased frequency of fractures of the pelvis and lower frequency of fractures of the tibia and fibula as well as knee injuries. Fractures of tibia and fibula, so prevalent in bumper injuries, in our study were only found in $28.57 \%$ and $20 \%$ respectively, similar to the study of Starnes et al. ${ }^{1}$ which shows a percentage of tibial fractures of $27.3 \%$. On the other hand in the study of Schmucker ${ }^{2}$ these fractures appear in $40.54 \%$ of cases, but it recognizes the need to further investigate the mechanism of fracture of tibia and fibula since in many cases these lesions appear on the contralateral leg. Thus, the mechanism of injury is not completely identified so far.

The most common chest injuries where rib fractures, a fact that coincides with the study of Schmucker et $\mathrm{al}^{2}$. The major abdominal injuries in this study were in liver and spleen. In our study, however, although liver lesions are the most frequent, the spleen ones are more infrequent, appearing only in $8.5 \%$ of cases.

The ISS average found in the study of Peng et al. ${ }^{12}$ was $24.5 \pm 1.0$ in dead people, which is 2 units lower than in our study, while in the research performed by Schmucker et al. ${ }^{2}$ was 31 , Schmucker acknowledges that the ISS is in the upper limit compared to other studies (9-34) since they have excluded cases produced at a speed under 20 $\mathrm{km} / \mathrm{h}$. In our study, it is also reasonable that the ISS was observed at the upper limit since only have been studied cases resulting in death, so that injuries are more severes and therefore it is logical to expect an ISS greater. Also in this same study Schhucker et al. ${ }^{2}, 62 \%$ had a $4+$ MAIS and in our study this percentage was $60 \%$.

From ISS 9-15 there is a total of five pedestrians (14\%) that are considered moderately injured patients with result of fatality. In four of the cases the possible explanation of the fatality is due to the limitation of the ISS. For the four cases all the AIS3 injuries are located in the head and therefore only one AIS3 is considered for the ISS calculation. 
In the case of using the NISS the values are increased to 22-27 due to the multiple anatomical region consideration.

A current limitation of our study is the lack of quantification of injuries of pedestrians hit by motorcycles when pedestrian does not die as a result of the injuries or further complications, which can explain the higher ISS score and the lower incidence of injuries in lower limbs. The lower incidence of fractures of the tibia and fibula in our study could also be due to the autopsy technique itself, since during the external examination of the body, a closed and non-displaced fracture from one of the two leg bones may be unnoticed, when there is not any deformity or joint mobility due to postmortem rigor mortis phenomenon, although this phenomenon is partly minimized by the fact that most of the fatally injured pedestrians were previously treated in clinical settings, where these injuries could be easily seen by radiological study, and our study incorporated also the medical report.

Another limitation, commonly present in the field of research related to pedestrian protection, is the difficulty to distinguish between the injuries produced by first and/or second impact; additional investigations are needed to clarify this topic.

\section{Conclusions}

A retrospective descriptive study of pedestrian's deaths after collisions with motorcycles in an urban area like Barcelona was conducted; the conclusion of this investigation shows the injury pattern of fatal motorcycle-pedestrian collisions. The main injuries sustained by the pedestrian were located in head, thorax and lower extremities. In the case of the head injuries, the subarachnoid hemorrhage in $71.4 \%$ of cases, cerebral contusions and skull base fractures (65.7\%) and subdural hematomas (54.3\%) were the most frequent injuries while the most frequent thoracic injury were rib fractures (45.7\%), followed by pelvic and tibia fractures (28.6\%). Traumatic brain injury was the main cause of death determined in the autopsy found in $62.85 \%$ of the cases, followed by hypovolemic shock in $20 \%$ of the cases, and $5.7 \%$ due to a combined thoracic and brain trauma.

There is a need to increase the research involving accidents with motorbikes, the kinematics of the collision, the energies involved and areas of the vehicle responsible 
for different injuries, since the behavior of the impact from structure of the motorcycle is unknown to date. Due to the differences in the injury pattern and the number of detected fatal accidents, it would be advisable that the European mandatory measures provided for the design of $\mathrm{M} 1$ vehicles for pedestrian protection were explored and properly applied in the future to other vehicles, such as the motorcycles, because has been proved that motorcycle is a vehicle with the potential of inflict serious injuries to the pedestrians in case of collision.

\section{Bibliography}

1. Starnes MJ, Hadjizacharia P, Chan LS, et al. Automobile versus pedestrian injuries: Does gender matter? J Emerg Med. 2011;40:617-22

2. Schmucker $U$, Beirau M, Frank $M$, et al. Real-world car-to-pedestrian-crash data from an urban centre. J Trauma Manage Outcomes. 2010;4:1-6

3. Damsere-Derry J, Ebel BE, Mock CN, et al. Pedestrian Injury Patterns in Ghana. Accid Anal Prev. 2010;42:1080-8

4. World health Organization (WHO) (2013) Pedestrian safety: a road safety manual for decision makers and practitioners. Geneva, Switzerland: World Health Organization (132 pp.)

5. World health Organization (WHO) (2009) Global status report on road safety. Time for action. Geneva, Switzerland: World Health Organization (287 pp.)

6. Chalya PL, Mabula JB, Dass RM, et al. Injury characteristics and outcome of road traffic crash victims at Bigando Medical Centre in Northwestern Tanzania. J Trauma Manage Outcomes. 2012;6:1-8 
7. Arregui-Dalmases C, Fredriksson R, Kerrigan J, Velazquez-Ameijide J, SanchezMolina D. Pedestrian protection, overview. John Wiley \& Sons Ltd editors. Encyclopedia of Automotive Engineering. ISBN: 978-0-470-97402-5. pp. 3049-3072.

8. Solagberu BA, Ofoegbu CKP, Nasir AA, et al. Motorcycle injuries in a developing country and the vulnerability of riders, passengers, and pedestrians. Inj Prev. 2006;12:266-8

9. Gisbert JA, Verdú FA. Accidentes de tráfico. En: Gisbert JA. Medicina Legal y Toxicología. $5^{\text {a }}$ ed, reimpr: Masson; 1998. p. 336-348.

10. Arregui-Dalmases C, Luzón Narro J. Protección de peatones. Fundamentos de Biomecánica en las lesiones por accidente de tráfico (biomechanics fundaments in traffic injury). Editorial ETRASA, ISBN: 978-84-92625-40-6. Pages: 315-336, 2011.

11. Siram SM, Sonaike V, Bolorunduro OB, et al. Does the Pattern of Injury in Elderly Pedestrian Trauma Mirror That of the Younger Pedestrian? J Surg Res. 2011;167:14-8

12. Peng RY, Bongard FS. Pedestrian Versus Motor Vehicle Accidents: An Analysis of 5,000 Patients. J Am Coll Surg. 1999;189:343-8

13. Ehrlich E, Tischer A, Maxeiner H. Lethal pedestrian-passenger car collisions in Berlin Changed injury patterns in two different time intervals. Legal Medicine. 2009;11:324-6

14. Arregui-Dalmases C, Lopez-Valdes FJ, Segui-Gomez M. Pedestrian injuries in eight European countries: An analysis of hospital discharge data. Accident Anal Prev. 2010;42:1164-71.

15. Dandona R, Kumar GA, Raj TA et al. Patterns of road traffic injuries in a vulnerable population in Hyderabad, India. Injury Prevention. 2006;12:183-8 